\title{
Causação Cumulativa em Myrdal e seus Desdobramentos Enquanto Alternativas ao Conceito de Equilíbrio
}

Thiago Dumont Oliveira ${ }^{1}$

Luccas Assis Attílio

Resumo: O artigo discute em que medida o conceito de causação cumulativa (em geral) permite superar as dificuldades relacionadas ao conceito de equilíbrio para se pensar em desenvolvimento econômico e apresenta o conceito de causação cumulativa de Myrdal (em particular). Para além de argumentar que a abordagem de Myrdal consiste em um arcabouço amplo que incorpora elementos pós-keynesianos, institucionalistas e evolucionários, analisamos alguns trabalhos de Setterfield como um desdobramento do pensamento de Myrdal. Nosso argumento é de que através de uma visão holística do sistema econômico que combina distintas vertentes, pode-se tratar o tema desenvolvimento econômico combinando questões relacionadas ao crescimento econômico com a análise histórica da evolução das instituições. Para ilustrar discutimos a incorporação de path dependency, lock-in e institutional interrelatedness em um modelo proposto por Setterfield.

Palavras-Chave: Myrdal, Veblen, Kaldor.

JEL: B52, O11, O43 


\section{Cumulative causation in Myrdal and it's unfoldings as alternatives to equilibrium}

Abstract: This paper assesses the extent to which the concept of cumulative causation (in general) overcomes the shortcomings of equilibrium analysis to address issues related to development economics and presents Myrdal's concept of cumulative causation (in particular). Furthermore, we claim that Myrdal's broad approach comprises Post-Keynesian, Institutionalist and Evolutionary elements and analyse some works of Setterfield as an extension of Myrdal's conception. Our point is that through a holistic approach to Economics that combines post-keynesian, institutional and evolutionary elements it is possible think about development beyond growth, accounting also for the historical evolution of institutions. To illustrate how this can be accomplished we present a model by Setterfield in which path-dependency, lock-in and institutional interrelatedness are included.

Key-Words: Myrdal, Veblen, Kaldor.

JEL: B52, O11, $\mathrm{O}_{43}$

\section{Introdução}

O conceito de equilíbrio permeia a história do pensamento econômico desde Adam Smith. Raros são os economistas que se aventuraram em pensar a ciência partindo de outra base, entre eles alguns nomes mais conhecidos são Marx, Schumpeter, Veblen, Myrdal, Young e Kaldor. Além desses autores, há outros que recebem menos atenção como Patinkin, Clower e Leijonhufvud que nas décadas de 60 e 70 lançaram uma agenda de pesquisas desequilibrista virtualmente esquecida pelos economistas contemporâneos.

Esse movimento desequilibrista surgiu como uma resposta à síntese neoclássica de meados do século XX em que o arcabouço Walrasiano foi conciliado com o Keynesiano, inaugurando a era do equilíbrio geral enquanto modus operandi da ciência. $\mathrm{O}$ ceticismo em relação à síntese neoclássica originou um período de intenso debate entre economistas a partir da década de 1970 A ciência econômica tornou-se um campo de guerra até a década de 9o, que veria a emergência de um novo paradigma. O consenso entre os Fresh Water e os Salt Water na virada do século, ou a nova síntese neoclássica, lançou a teoria do equilíbrio geral em novas bases: os modelos DSGE. Assim, nota-se a resiliência do conceito de equilíbrio na história do pensamento econômico.

É curioso notar que entre os autores que destacamos como críticos à ideia de equilíbrio há um considerável horizonte temporal, iniciando com Marx em meados do século XIX e se estendendo até a década de 1970; nesse sentido todas as tentativas de tirar a ciência econômica de seu próprio equilíbrio mostraram-se frustradas, como se houvesse uma força que atrai os economistas novamente para o mesmo pressuposto a cada choque metodológico recebido.

Apesar da supremacia do conceito de equilíbrio na história do pensamento econômico, o presente artigo analisa as dificuldades inerentes a esse conceito para se pensar em desenvolvimento econômico, apresentando o conceito de causação cumulativa de Myrdal como alternativa. Mais especificamente, nosso argumento é composto de duas partes. A primeira parte do argumento apresenta uma extensão da interpretação de Fujita (2004; 2007). Fujita mostra que o conceito de causação cumulativa de Myrdal pode ser interpretado como uma conciliação do pensamento de Young/Kaldor e Veblen. Não obstante, o artigo não explicita as vantagens do conceito de causação cumulativa para se pensar em desenvolvimento econômico vis-à-vis os problemas teóricos relacionados ao conceito de equilíbrio. Portanto, o primeiro objetivo do trabalho consiste em identificar os limites do conceito de equilíbrio e em que medida a abordagem da causação cumulativa se mostra mais propícia para se pensar em desenvolvimento econômico, ressaltando a visão vebleniana no que tange às mudanças institucionais. Essa primeira parte, portanto, motiva a segunda parte em que discutimos o conceito de causação cumulativa de Myrdal e desdobramentos deste. Em particular, avaliamos alguns trabalhos recentes de Setterfield $(1993 ; 1997 \mathrm{a} ; 1997 \mathrm{~b})$ que tratam o desenvolvimento econômico a partir dessa perspectiva ampla a la Myrdal; a intenção é mostrar em que medida o conceito de causação cumulativa de Myrdal é passível de formalização através dos trabalhos desse autor. Esperamos assim contribuir para uma maior interpenetração entre os trabalhos de pós-keynesianos, institucionalistas e economistas evolucionários que ainda se encontra incipiente.

O trabalho, além dessa introdução, está estruturado em cinco seções. A segunda seção discute os problemas relacionados à ideia de equilíbrio e o conceito de causação cumulativa de Kaldor. A terceira seção ressalta a importância das instituições para se pensar em desenvolvimento econômico. A quarta seção apresenta o conceito de causação cumulativa em Myrdal e em que medida ele fornece um arcabouço amplo que une preceitos pós-keynesianos, institucionalistas e evolucionários. Na quinta seção avaliamos alguns trabalhos de Setterfield enquanto desdobramentos da agenda de pesquisas de Myrdal a fim de ilustrar como esse conceito desenvolvido por Myrdal é passível de formalização. Na sexta seção tecemos considerações finais. 


\section{Dificuldades Inerentes ao Conceito de Equilíbrio e Cau- sação Cumulativa Enquanto Alternativa}

Kaldor (1972) ressalta em que medida a ideia de equilíbrio é um obstáculo para o desenvolvimento da economia como ciência. A crítica de Kaldor (1972) é especificamente à teoria do equilíbrio geral iniciada por Walras e levada adiante por uma série de economistas matemáticos dos quais Debreu é o mais proeminente. A teoria de Debreu, dirá o autor, não explica como os preços são de fato determinados; tem apenas caráter lógico no sentido de que pode ser deduzida a partir de hipóteses. Seu objetivo é determinar o mínimo de hipóteses que garantam um conjunto de preços de equilíbrio que são: i) únicos, ii) estáveis e iii) satisfazem as condições de Pareto otimalidade.

A teoria do equilíbrio geral refere-se a um tipo particular de modelo:

[M]icroeconomic in that it modeled an arbitrary number of potentially heterogeneous agents, but general in that it modeled all markets simultaneously and that very general assumptions were made about consumers' preferences and about technology. Moreover, general equilibrium analysis focused on proving that equilibrium existed and investigating whether it was unique and stable (Backhouse e Boianovsky 2014: p. 9).

Kaldor (1972) argumenta que essa teoria, embora não vise a descrever a realidade, é entendida como pré-condição para explicar de forma lógica e consistente como um sistema descentralizado funciona assegurando satisfação máxima para a sociedade nos termos de Pareto. Seu ceticismo em relação a essa agenda de pesquisa é inequívoco: "[W]ithout a major act of demolition - without destroying the basic conceptual framework - it is impossible to make any real progress" (ibid: 1240). Kaldor identifica o quarto capítulo de "A Riqueza das Nações" (Smith 1996 [1776]) como o momento chave na introdução do conceito de equilíbrio na ciência econômica:

One can trace a more or less continuous development of price theory from the subsequent chapters of Smith through Ricardo, Walras, Marshall, right up to Debreu and the most sophisticated of present-day Americans (Kaldor 1972: 1241).

A partir desse capítulo a teoria do valor torna-se central e sua obra passa a focar nos aspectos alocativos e desconsidera a função criativa dos mercados enquanto um instrumento para promover mudanças econômicas. Conforme veremos ao longo do artigo,

models of cumulative causation represent an effort to refocus growth theory on the problem of creating productive resources over time (rather than allocating given productive resources at a point in time), treating the heritage of the past as the only truly given variable in the system (Setterfield 1997a: 366).

A ideia de equilíbrio pressupõe custos constantes e retornos constantes. Em Smith e Ricardo isso implica precos naturais determinados unicamente pelos custos de produção. Para os neoclássicos a hipótese de função de produção linear e homogênea é um pré-requisito para que a ideia de maximização dos lucros e competição perfeita sejam consistentes. Marshall tentou acomodar retornos crescentes e decrescentes, mas Sraffa (1926) demonstrou a impossibilidade disso.

Kaldor (1935) mostrou que a livre entrada de empresas em um cenário de competição imperfeita não necessariamente leva à seleção das empresas mais adaptadas, podendo ter o efeito adverso de criar capacidade ociosa e aumentar os custos das empresas participantes em vez de reduzir os preços. Esse aumento de custos está relacionado à diminuição da eficiência técnica que deriva da diminuição da produção de cada participante e, consequentemente, da incapacidade de absorver os ganhos de escala. Portanto, Kaldor (1935: 33) argumenta que a livre entrada de empresas não garante que a economia convergirá em equilíbrio para um mercado perfeitamente competitivo, pois a "doctrine of 'excess capacity' [...] admits possibilities which the traditional 'laws of economics' seem to have excluded: e.g. that an increase in 'supply' may be followed by a rise in price". Além disso, as empresas possuem "institutional monopolies" que inviabilizam a hipótese de que seus produtos sejam substitutos perfeitos; diversas variáveis fazem com que os produtos não sejam idênticos, e.g., o nome da firma, o costume do consumidor em comprar de certo vendedor e a distância da firma (Kaldor 1935: 44).

Sweezy (1939) argumenta que as curvas de demanda não podem ser utilizadas para analisar oligopólios, pois elas são construídas presumindo que à medida que uma empresa modifica seu preco as empresas concorrentes não reagirão alterando também seus preços. Todavia, a decisão do empresário é pautada em uma curva de demanda formada pelas expectativas em relação às prováveis reações de seus concorrentes. Deste modo, abandonado esse construto, o autor argumenta que a igualdade entre receita marginal e custo marginal definindo a situação de equilíbrio tem pouca relevância prática:

Generally speaking, there may be any number of price-output combinations which constitute equilibrium in the sense that, ceteris paribus, there is no tendency for the oligopolist to move away from them. But which of these combinations will be actually established in practice depends upon the previous history of the case. Looking at the problem in this way the theorist should attempt to develop an analysis which will enable him to understand the process of change which characterize the real world rather than waste his time in chasing the will-o'-the-wisp of equilibrium (Sweezy 1939: 573). 
Hall e Hitch (1939), na mesma esteira, utilizaram questionários para analisar a decisão de empresários quanto a preços e nível de produção. Os autores concluem que os empresários não tomam suas decisões tentando igualar receita marginal e custo marginal; os agentes usam uma "rule of thumb" e não ajustam preços se as mudanças de custos são percebidas como temporárias (Hall e Hitch 1939: 18). Assim a história de cada indústria é um elemento que influencia a decisão dos empresários em relação a preços e nível de produto:

The 'current doctrine' of the equilibrium of the firm [...] breaks down in [...] oligopoly and monopolistic competition with oligopoly; these, as special cases, are relegated to footnotes or left to mathematicians, because the demand curve for the product of the individual firm, and therefore marginal revenue, is indeterminate where the price and output policies of the firms are interdependent (Hall e Hitch 1939: 17).

Robinson (1953) argumenta que o empresário não tem como objetivo último maximizar o lucro no curto prazo. Se uma determinada ação é rentável, mas prejudicial à reputação da empresa, ele optará por um curso menos rentável que não coloque em risco suas ações futuras. Ademais, a autora critica a ideia de que existe um nível normal de lucro associado ao tamanho ótimo da firma em equilíbrio. Se os custos de expansão de uma empresa em um oligopólio não compensam o retorno esperado, essa pode expandir para novos mercados. Em relação à escolha de preço e nível de produção, Robinson argumenta que em teoria é possível descrever um sistema de equações simultâneas para resolver o problema da maximização do lucro, mas na prática o empresário não tem dados suficientes para resolver o problema. Além disso, mesmo se tivesse dados suficientes ele não teria capacidade cognitiva para resolver tal problema em tempo hábil, dado que o mercado está sujeito a alterações constantes. Finalmente, a autora ressalta a importância de incluir o elemento temporal na análise, e, embora não use a expressão path-dependency, seu argumento vai ao encontro deste conceito:

It is only in a metaphorical sense that price, rate of output, wage-rate or what not can move in the plane depicted in a price-quantity diagram. Any movement must take place through time, and the position at any moment of time depends upon what it has been in the past $[. .$.$] any adjustment takes a certain time to complete and$ [...] events may occur meanwhile which alter the position, so that the equilibrium towards which the system is said to be tending itself moves before it can be reached [...] the very process of moving has an effect upon the destination of the movement, so that there is no such thing as a position of long-run equilibrium which exists independently of the course which the economy is following at a particular date (Robinson 1953: 90).

Os teóricos do equilíbrio geral desconsideraram esses problemas em sua formulação abstrata em que o ambiente independe da história, sem path-dependency, em que a passagem do tempo é virtual:

The very notion of 'general equilibrium' carries the implication that it is legitimate to assume that the operation of economic forces is constrained by a set of exogenous variables which are 'given' from the outside and stable over time (Kaldor 1972: 1244).

Para além disso, a ideia de equilíbrio geral assume que as instituições não desempenham qualquer papel e que independente da situação inicial o sistema irá convergir para um único ponto. Embora Kaldor mencione a importância das instituições, será Myrdal que, sob a influência de Veblen, vai incorporar as instituições como peças centrais de sua teoria.

A crítica de Kaldor à ideia de equilíbrio está apoiada na ideia de retornos crescentes de escala. Com a introdução de retornos crescentes de escala, as forças que levam a mudanças contínuas passam a ser endógenas e se propagam de forma cumulativa, cada período não pode ser previsto a não ser pela sequência de eventos que o antecederam.

A concepção de causação cumulativa de Kaldor é inspirada em Young (1928), que argumentou que a divisão do trabalho depende do tamanho do mercado e vice-versa, pois ao menos potencialmente cada bem produzido possibilitaria o surgimento de outros bens. Portanto, a divisão do trabalho depende da divisão do trabalho; esta não é uma conclusão tautológica, mas uma visão processual do sistema econômico em que os efeitos de retroalimentação são levados em conta. "It means that the counter forces which are continually defeating the forces which make for economic equilibrium are more pervasive and more deeply rooted than we commonly realize" (Kaldor 1972: 1245).

Pré-condição para mudança cumulativa é que o aumento da oferta de um bem aumente a demanda por todos os outros bens:

[T]he process of economic development can be looked upon as the resultant of a continued process of interaction - one could almost say, of a chain-reaction - between demand increases which have been induced by increases in supply, and increases in supply which have been evoked by increases in demand (Kaldor 1972: 1246).

Sumarizando a teoria da causação cumulativa de Kaldor (1966), o crescimento produtivo do setor manufatureiro induz ao crescimento da produtividade desse setor através dos retornos crescentes de escala. Concomitantemente, haverá a transferência de trabalho do setor não manufatureiro para o setor manufatureiro, o que implica crescimento da produtividade da economia. Consequentemente, a competitividade das exportaç̃es ampliará se o país aderir à estratégia export-led. Por fim, $\mathrm{o}$ aumento das exportaç̃es expande a produção do setor manufatureiro, fechando o círculo virtuoso e gerando um processo de causação cumulativa.

Revista de Economia, v. 40, n. 3 (ano 38), p. 28-46, set/dez. 2014. Editora UFPR 
Embora haja pontos de contato entre Kaldor e $\mathrm{Myrdal}^{1}$, as diferenças são também notáveis. Antes de apresentar o conceito de causação cumulativa de Myrdal, discutimos brevemente a centralidade das instituições na tradição vebleniana.

\section{Causação Cumulativa em Veblen e a Relevância das}

O institucionalismo se caracteriza pela análise das mudanças institucionais e da relação mútua entre o indivíduo e a estrutura social. Uma das críticas dos institucionalistas à economia neoclássica consiste na necessidade de se estabelecer um diálogo com a Sociologia, posto que as atividades econômicas são indissociáveis da esfera social (Lewin 1996). Nessa esteira, Veblen (1898: 377-378) clama por "theories of a comprehensive process by the notion of a cumulative causation". Para Veblen, o ambiente não pode ser reduzido à esfera econômica, sua intenção era internalizar os hábitos em sua concepção de crescimento, e, nesse sentido, a cultura se torna central para compreender o processo econômico (Argyrous e Sethi 1996). De acordo com esses autores, a teoria evolucionária não se resume a uma teoria dinâmica em contraposição a uma teoria estática. A teoria dinâmica e processual é uma condição necessária, mas não suficiente para uma teoria evolucionária. O ponto central de uma teoria evolucionária é evitar a teleologia.

O caráter evolucionário que Veblen reivindica não assume o sentido de evolução para algo necessariamente melhor. No bojo de sua crítica ao caráter teleológico da economia neoclássica reside a ideia darwiniana de que a evolução pode tomar múltiplos caminhos e que não faz sentido pensar em um fim específico para o processo evolutivo. Assim, o indivíduo e a sociedade se relacionam de forma circular e cumulativa, se afetam mutuamente, e as instituições são o intermédio dessas relações.

Para Veblen, uma ciência evolucionária é uma teoria do processo; a análise da sequência de eventos. O cientista evolucionário, dirá o autor, carrega uma atitude espiritual que o distingue do cientista não evolucionário, que tende a buscar respostas em termos de causa e efeito. "The ultimate term in their systematization of knowledge is a 'natural law"' (Veblen 1898: 378).

A concepção hedonística do homem associada à formulação de leis naturais tem como base um agente que responde a estímulos impulsionado por sua busca por prazer, mas que não é afetado pelas instituições: "He has neither antecedent nor consequent. He is an isolated, definitive human datum, in 1 Eles escreveram aproximadamente na mesma época, tinham como foco a economia internacional e usaram dados de forma recorrente (Fujita 2004: 10). stable equilibrium" (Veblen 1898: 389). Para Veblen, portanto, o caráter evolucionário da ciência econômica consiste na compreensão dos métodos que os homens utilizam para lidar com as condições materiais mundanas; enquanto as propriedades físicas dos materiais a que o homem tem acesso são constantes, é o homem que muda constantemente em sua percepção de como utilizar esses materiais. Assim, o indivíduo deve ser compreendido a partir do processo cumulativo de adaptação dos meios aos fins, em qualquer ponto do tempo suas ações só podem ser entendidas à luz dos eventos passados que originaram a situação presente.

Conforme Neale (1987) explica, a análise institucional pressupõe que a maior parte das ações individuais sejam influenciadas pelas instituiç̃oes. Não obstante, isso não quer dizer que os indivíduos perdem sua autonomia, mas que suas ações não ocorrem in vacuo e são ininteligíveis se se perder de vista as instituições:

[C]ulture defines the permissible and forbidden, defines right and wrong, the admirable and its opposite, gives content to these definitions with rules for behavior, and so provides opportunities as well as limits (Neale 1987: 1179).

Importante destacar que os institucionalistas da tradição vebleniana distinguem o duplo papel das instituições. Essa dicotomia instrumental/cerimonial implica que de um lado há um caráter instrumental que deriva da busca dos agentes por novas soluções para os problemas presentes, mas, simultaneamente, as instituições são "past-binding” no sentido de que as crenças e costumes em um dado momento derivam de experiências passadas (Neale 1987: 1197). Essa dicotomia não é uma contradição, mas duas faces da mesma moeda: "Institutions change within the context of existing rules and folkviews, adapting some rules and creating some new ones" (Neale 1987: 1200).

\section{Causação Cumulativa em Myrdal e a Conciliação de Veblen e Kaldor}

Diante das duas versões de causação cumulativa apresentadas, pode-se argumentar que a concepção de Myrdal se caracteriza como a justaposição dessas abordagens. Fujita (2004; 2007) propõe que Myrdal deve ser compreendido como a apoteose do conceito de causação cumulativa para se pensar em desenvolvimento, embora geralmente seja atribuída a Kaldor essa posição. $\mathrm{O}$ autor argumenta que não se trata de fazer um juízo de valor e alegar a supremacia de Myrdal sobre Kaldor, mas, antes, apontar a incorporação de elementos veblenianos por parte de Myrdal que levam em outras direções, podendo ambas as análises se complementar. 
Primeiro ponto interessante de Myrdal, essencial para qualquer discussão sobre desenvolvimento econômico, é que ele distingue crescimento e desenvolvimento, em que desenvolvimento significa ascensão social de toda a população de determinado país (Myrdal 1977).

O conceito de causação cumulativa de Myrdal, teoria que é a base de seu pensamento no tocante ao desenvolvimento tanto de países como de regiões, "envolve, naturalmente, uma constelação circular de forças, que tendem a agir e a reagir independentemente" (Myrdal 1960: 27).

O que o autor enfatiza ao assinalar a circularidade das forças, é que um pequeno choque em determinada variável é capaz de gerar efeitos em todo o sistema e que a variável que causou o choque inicial pode vir a sofrer efeitos advindos das outras variáveis nas quais essa variável inicial exerceu o seu efeito. Por exemplo, uma das conclusões do estudo de Myrdal (1996 [1944]) sobre a desigualdade racial nos Estados Unidos da América é de que a pobreza dos negros é exacerbada pelo preconceito dos brancos ao mesmo tempo em que a pobreza auxilia no enraizamento do preconceito. Em suma, a pobreza seria a causa e consequência da situação desprivilegiada dos negros.

Outro ponto que merece atenção, no mesmo espírito das seções anteriores, é que Myrdal não concorda com a tese de equilíbrio estável, o que pode ser interpretado como um corolário da causação circular: "O sistema não se move, espontaneamente, entre forças, na direção de um estado de equilíbrio, mas, constantemente, se afasta dessa posição" (Myrdal 1960: 28)

$\mathrm{O}$ conceito de causação cumulativa de Myrdal em sua fase final consiste em quatro aspectos (Fujita 2004: 7-8): i) A tese dos backwash effects visa a explicar a ampliação do hiato entre países e regiões desenvolvidas e em desenvolvimento, ii) A tese dos spread effects apresenta uma lógica de convergência, iii) $\mathrm{O}$ terceiro aspecto se relaciona ao segundo; embora cético em relação à possibilidade da convergência ocorrer de forma natural, Myrdal via nas políticas governamentais um papel importante no sentido de buscar maior igualdade entre os países e iv) O último aspecto de sua obra é a incorporação de elementos veblenianos, ou seja, a importância dos fatores institucionais e não econômicos ao escopo da análise. Cabe descrever cada um desses aspectos brevemente.

O conceito de backwash effects (Myrdal 1960) explana que quando um centro regional recebe um impulso econômico, as outras regiões em sua volta podem ser prejudicadas, uma vez que variáveis como a migração, os movimentos de capital e o comércio tenderiam a favorecer regiões mais desenvolvidas em detrimento das regiões mais atrasadas. Os indivíduos buscariam os centros com maiores oportunidades de emprego e renda. Os investimentos, da mesma forma, fluiriam onde percebessem dinamismo econômico. Portanto, expansão do comércio nas regiões mais avançadas teria como contraparte diminuição do comércio nas partes menos avançadas, o que aprofundaria a disparidade regional. Juntos, esses fatores alargariam o hiato entre regiões e países desenvolvidos em relação aos em desenvolvimento.

É importante se ater ao fator migratório na análise desse problema, pois não se trata de uma variável "econômica". Nota-se, deste modo, um diálogo com as ideias de Veblen (1898), que seriam cristalizadas de forma mais contundente na incorporação de diversos fatores "não-econômicos" para entender o desenvolvimento, que será analisado em breve.

Myrdal se aproxima de Kaldor (1972) no que tange aos spread effects. Ao contrário dos backwash effects, esse fator acarretaria a convergência de países e regiões atrasadas com as partes avançadas. Nessa concepção, o trabalho de Young (1926) também se estreita com o de Myrdal, na medida em que Young traç um paralelo entre o dinamismo do mercado e suas especificidades conforme explicado na segunda seção. Myrdal (1960), de forma análoga, parte do mesmo pressuposto.

Todavia, os spread effects podem causar trajetórias divergentes para as regiões e países. De acordo com Myrdal (1960), não somente as desigualdades regionais são maiores nos países pobres, como também ao passo que essas desigualdades se reduzem nos países ricos, elas crescem nos países pobres. Deriva dessa tese a descrença de Myrdal na força do livre mercado para promover o desenvolvimento. "O processo acumulativo, quando não controlado, promoverá desigualdades crescentes” (Myrdal 1960: 27).

Assim, o terceiro aspecto do pensamento de Myrdal consiste no uso do instrumental governamental para mitigar e retirar os países e regiões em desenvolvimento da situação de paulatina deterioração econômica, social e política. Myrdal (1977) vislumbra as políticas públicas como fator contra-cíclico da tendência desses países retardatários a continuarem na trajetória de subdesenvolvimento. Após a Segunda Guerra Mundial, o autor percebe os Estados de Bem-Estar Social como exemplos de instituições que podem melhorar o padrão de vida de sua população, e, portanto, recomenda que as nações subdesenvolvidas sigam esse caminho.

Em relação aos países subdesenvolvidos, Myrdal (1977) defende profundas reformas igualitárias, que podem ser entendidas por reformas agrárias, estabelecimento de impostos progressivos, ampliação da democracia, entre outras. Subjacente a isso, novamente a influência de Veblen (1898) se faz presente, pois essas reformas defendidas são justificadas no intuito de tirar essas nações dos ciclos viciosos (path-dependent) em que se encontram.

Por fim, destaca-se a inclusão de fatores "não-econômicos" na análise do desenvolvimento: "Todos os fatores 'não-econômicos' - estrutura política, social, e econômica, instituições e atitudes, enfim, todas as relações interpessoais - têm que ser incluídas na análise" (Myrdal 1977: 26). O uso dessas variáveis contrasta com a modelagem padrão que corriqueiramente é utilizada 
por economistas. Deste modo, Myrdal (1977) critica a ênfase em modelos matemáticos e o alastramento de conceitos e teorias que podem ser adequados para nações desenvolvidas, mas que pouco explicam a situação dos Estados subdesenvolvidos.

Ao se aproximar de Veblen devido à maior importância concedida a fatores históricos, culturais e institucionais, Myrdal analisa a dinâmica do desenvolvimento em países atrasados sob uma diferente perspectiva. Por outro lado, embora sua tese dos backwash effects não encontre contrapartida em Kaldor, sua tese dos spread effects dialoga com este autor.

Portanto, Myrdal pode ser interpretado como uma ponte entre o trabalho de pós-keynesianos, institucionalistas e evolucionários, oferecendo uma análise que incorpora tecnologia, produção, distribuição e demanda efetiva de um lado e a evolução das instituições e sua interação com fatores econômicos de outro. Curiosamente, Argyrous e Sethi (1996) chamam a atenção que fatores históricos e culturais permanecem como uma caixa preta entre os pós-keynesianos, apesar de alegações como a de Kalecki que "the long-run trend is but a slowly changing component of a chain of short-period situations; it has no independent entity" (1971: 165). Não obstante, embora o legado de Kaldor seja consideravelmente mais amplo que o de Myrdal, a conciliação entre elementos pós-keynesianos, institucionalistas e evolucionários a la Myrdal mostra-se uma agenda de pesquisas promissora. Ilustraremos esse ponto através de alguns trabalhos de Setterfield, todavia há outros autores que seguiram essa linha(e.g. Cornwall e Cornwall 1987, Nell 1988; 1992, Hodgson 1989 e Cornwall 1990).

\section{Desdobramentos de Myrdal: Setterfield}

Setterfield (1993; 1997a; 1997b) é um dos autores a tratar o processo de causação cumulativa em um espírito semelhante a Myrdal. Em seu artigo de 1993, que é tratado com mais detalhes no seu livro (1997b), Setterfield introduz hysteresis em seu modelo de causação cumulativa:

Hysteresis exists when the long-term value of a variable depends on the value of the variable in the past, by virtue of the influence of this past value on the alleged exogenous variables, coefficients, and structural equations that characterize the system that determines the variable. In an institutional context, hysteresis will exist when current institutions influence the nature of current economic activity, which in turn influences subsequent institutional forms (Setterfield 1993: 760).

Sua intenção é formular um modelo que supere tanto o velho institucionalis- mo quanto o novo institucionalismo, pois o autor julga ambas as abordagens insatisfatórias. Os velhos institucionalistas tratam a economia de maneira holística, o todo não pode ser compreendido pela análise de suas partes, e tem na análise histórica seu principal ferramental. Isso implica uma ênfase nas estruturas (relações entre indivíduos e instituições) e não nas ações dos indivíduos (escolhas). Por outro lado, os novos institucionalistas têm como foco o auto interesse dos indivíduos, argumentando que as instituiç̃es aparecem espontaneamente como consequência da interação entre indivíduos racionais maximizadores que valorizam essas instituições.

The emphasis in the NIE [New Institutional Economics] is on 'invisible hand'mechanisms; it is argued that interaction between rational individuals produces social patterns of behavior without any one individual directing the results (Setterfield 1993: 758).

Destarte, Setterfield (1993) critica o caráter estruturalista do velho institucionalismo e o fato de que as instituições são endógenas no novo institucionalismo. $\mathrm{O}$ autor propõe uma abordagem dinâmica que busca conciliar as duas visões, tal que o comportamento individual molda o surgimento de novas instituições ao mesmo tempo em que o comportamento individual é moldado pelas instituições já existentes, ou seja, um processo com hysteresis.

Setterfield combina as duas visões ao colocar as instituições como exógenas no curto prazo, como restrições, mas endógenas no longo prazo, fruto das ações dos indivíduos e sujeitas a path-dependency. Trata-se de pensar em um processo dinâmico com efeitos de retroalimentação:

[S] uppose initially that the choice sets of individuals and groups are defined by currently existing institutions. These institutions will consequently exert a straightforward influence on current economic activity. This activity will in turn give rise to economic outcomes, which may be judged by individuals and groups as either good or bad. Depending on this evaluation, individuals and groups will then attempt to either modify existing institutional arrangements or retain the status quo, which will in turn affect the form of future institutions and hence future choice sets and activities, and so on (Setterfield 1993: 760-761).

Para além disso, o autor discute um processo de causação cumulativa que não tende a um equilíbrio eficiente de longo prazo, como advogam os proponentes do novo institucionalismo. Setterfield argumenta que não há de se falar em eficiência a longo prazo, posto que instituições não eficientes podem persistir indefinidamente no sistema econômico. Essas instituições podem permanecer em função de interesses econômicos de alguns atores que se beneficiam dessas instituições e estão em uma posição de influenciar na escolha dessas instituiç̃es. Há também a questão da "institutional interrelatedness" que pode explicar a permanência de instituições em função dos custos altos de 
reconfigurá-las independente de sua eficiência:

Interrelatedness refers to interconnections between components of the production process (machinery, human capital, organizational structures, etc.). These interconnections limit the possibilities for marginal adjustments to productive capacity (at a firm-, industryor economy-wide level) to those which conform to technological and institutional standards established in the past and embodied in existing productive assets (Setterfield 1997a: 372).

A economia pode então estar locked-in com relação a um tipo de instituição que se apresenta inferior a uma alternativa. Trata-se, portanto, de um caso clássico de path dependency amplamente discutido por economistas evolucionários (Setterfield 1993: 762-763).

Setterfield (1997a) formaliza os conceitos de path dependency e lock-in incorporando-os ao modelo de causação cumulativa de Kaldor, constituindo um desdobramento de Myrdal. Para tanto, o autor estende os modelos de Dixon e Thirlwall (1975), Thirlwall (1980) e McCombie e Thirlwall (1994) de forma a incorporar elementos históricos na análise do crescimento de longo prazo. Conforme argumentamos na seção anterior, duas teses centrais de Myrdal são os backwash effects e os spread effects. Esses dois conceitos têm importância para nosso argumento, pois admitem a possibilidade de um círculo virtuoso tornar-se um círculo vicioso ao longo do tempo (e vice-versa). Kaldor, por outro lado, não propôs nenhuma explicação de reversão de tendência de crescimento a longo prazo. A causação cumulativa em Kaldor tende a se propagar indefinidamente uma vez que o processo tenha se iniciado, $o$ que não se sustenta empiricamente ao se levar em consideração a ascensão e queda das grandes potências mundiais como discutido por Arrighi (1995). Para compreender como Setterfield lida com essa questão e estende a abordagem Kaldoriana para incorporar path dependency e lock-in, partimos de sua equação final derivada dos trabalhos supracitados ${ }^{2}$, que apresenta a taxa relativa de crescimento de um país:

$$
\frac{\dot{Y}_{j t}}{\dot{Y}_{w}}=\left(\lambda_{j} \alpha_{j} \beta_{j}\right)^{t} \frac{\dot{Y}_{j \mathrm{o}}}{\dot{Y}_{w}}+\lambda_{j}\left(\gamma_{j}-\alpha_{w} \beta_{j}\right) \sum_{i=0}^{t-1}\left(\lambda_{j} \alpha_{j} \beta_{j}\right)^{i}
$$

Em que Ý é a taxa de crescimento do produto,j é a região, w representa o resto do mundo, té o tempo, $\lambda$ é o coeficiente que expressa a taxa de crescimento do produto como função da taxa de crescimento das exportações, $\alpha$ é o coeficiente da Lei de Verdoorn que relaciona a taxa de crescimento da produtividade à taxa de crescimento do produto, $\beta$ é o valor absoluto da elasticidade preço da demanda em relação à diferença entre inflação do resto do mundo e a inflação de uma região e $\gamma$ é a elasticidade renda da demanda em relação à taxa de crescimento mundial.

Por questões expositivas suprimimos a manipulação algébrica para chegar a essa equação, que pode ser encontrada em Setterfield (1997a: $366-369$ )
Para compreender como Setterfield (1997a) expande o modelo de maneira a incorporar elementos evolucionários, suponha primeiro que $0<\lambda_{j} \alpha_{j} \beta_{j}<1$ Nesse caso, quando $t \rightarrow \infty$, temos que:

$$
\frac{\dot{Y}_{j t}}{\dot{Y}_{w}} \rightarrow \frac{\dot{Y}_{j}^{*}}{\dot{Y}_{w}}=\frac{\lambda_{j}\left(\gamma_{j}-\alpha_{w} \beta_{j}\right)}{1-\lambda_{j} \alpha_{j} \beta_{j}}
$$

Ou seja, a taxa de crescimento de longo prazo da região j relativamente ao resto do mundo tenderia a um estado de equilíbrio determinado unicamente por parâmetros exógenos ${ }^{3}$. Esse resultado não está de acordo com a ideia de causação cumulativa de Kaldor por dois motivos: i) A crítica desse autor sobre a relevância do equilíbrio conforme argumentamos na seção dois e ii) As condições iniciais não influenciam o crescimento de longo prazo. Por outro lado, se supusermos que $\lambda_{j} \alpha_{j} \beta_{j}>1$, a taxa de crescimento da região j relativamente ao resto do mundo é dada pela equação (1). Nesse caso, as condições iniciais voltam a ter importância e não há convergência para equilíbrio, ao espírito de Kaldor. Todavia, quando $t \rightarrow \infty, \dot{Y}_{j t} / \dot{Y} \rightarrow \infty$. Nesse sentido, teríamos um círculo virtuoso que se perpetuaria e não encontraria suporte histórico. Finalmente, se $\lambda_{j} \alpha_{j} \beta_{j}=1$, teríamos:

$$
\frac{\dot{Y}_{j t}}{\dot{Y}_{w}}=\frac{\dot{Y}_{j 0}}{\dot{Y}_{w}}+t \lambda_{j}\left(\gamma_{j}-\alpha_{w} \beta_{j}\right)
$$

Neste caso, o estado inicial volta a impactar o crescimento de longo prazo e não há convergência para equilíbrio, porém, quando $t \rightarrow \infty$ a taxa relativa de crescimento tenderá a ampliar de maneira perpétua caso $\lambda_{j}\left(\gamma_{j}-\alpha_{w} \beta_{j}\right)>0$ e vice-versa, novamente temos um cenário não plausível do ponto de vista empírico.

Podemos agora discutir como Setterfield expande o arcabouço Kaldoriano de forma a se aproximar de Myrdal. Note que o modelo acima descrito apenas considera o efeito das condições iniciais e elementos exógenos no crescimento de longo prazo. O modelo é, portanto, determinístico no sentido de que esses parâmetros tendem a se perpetuar, o que é insatisfatório por não incorporar fatores históricos que podem ajudar a explicar como um país que crescia muito passa a crescer pouco e vice-versa.

3 Como os parâmetros mudam a cada ponto do tempo, só haverá convergência para equilíbrio se a velocidade de ajuste for suficientemente rápida para garantir essa convergência. Esse não é um ponto central para o nosso argumento, masé in (versententel

Revista de Economia, v. 40, n. 3 (ano 38), p. 28-46, set/dez. 2014. Editora UFPR 
A fim de modelar a causação cumulativa como um processo histórico, Setterfield endogeniza os parâmetros a e $\gamma$ que eram tidos como exógenos. $O$ coeficiente de Verdoorn a mede o aumento do crescimento da produtividade em função do crescimento do produto a partir de retornos crescentes de escala. Esse coeficiente é influenciado por fatores intangíveis (learning by doing), bem como fatores tangíveis como certos tipos de capital fixo ou a presença de organizações específicas. Nesse sentido, a presença de institutional interrelatedness pode impedir a realização de retornos crescentes de escala impactando, portanto, o parâmetro $\alpha$. A presença de institutional interrelatedness pode configurar uma situação de lock-in em que um país herda uma técnica produtiva do passado e tem dificuldade de inovar, o que é conhecido entre economistas evolucionários como path-dependency. Portanto, um crescimento inicial rápido pode se reverter devido a esse fenômeno criando endogenamente as condições para uma diminuição do crescimento subsequente (Setterfield 1997a: 372).

Um raciocínio análogo se aplica ao parâmetro $\gamma$ que mede a elasticidade renda da demanda em relação ao crescimento mundial. À medida que a renda aumenta os consumidores mudam suas preferências, transitando por uma commodity hierarchy. Isso implica que conforme os países crescem, a demanda dos consumidores sofre alterações e é necessário adaptar a estrutura produtiva às novas preferências de forma a manter a competitividade internacional. Novamente uma região pode ficar locked-in em uma estrutura produtiva caso haja institutional interrelatedness e ter dificuldades para se adaptar às novas demandas, reduzindo assim o valor de $\gamma$ e, consequentemente, seu crescimento relativo (Setterfield 1997a: 372-373).

Em ambos os casos, Setterfield argumenta que a probabilidade de ocorrer lock-in é uma função crescente da taxa inicial de crescimento. Mais uma vez, foge ao escopo do presente artigo discutir detalhadamente o modelo; o ponto que queremos destacar é que o autor modela os dois parâmetros supracitados tornando-os endógenos (Setterfield 1997a: 374-376). Nesse sentido, ao encontro do nosso argumento, o autor revela a possibilidade de tratar o processo de causação cumulativa do ponto de vista histórico, desenvolvendo um modelo que permite tratar o crescimento de longo prazo como sujeito a variações e não como um movimento determinístico. Fica clara a conciliação de elementos pós-keynesianos, posto que seu modelo base é derivado de Thirwal e Kaldor-Verdoorn, e elementos institucionalistas e evolucionários, através dos conceitos de path-dependency, interrelatedness e lock-in.

Myrdal não formalizou suas ideias e, com efeito, embora em seus trabalhos iniciais ele acreditasse ser possível investigar a causação cumulativa através de uma série de equações, em trabalhos subsequentes ele desqualificou qualquer tentativa de abordar esse conceito do ponto de vista quantitativo, argumentando que a causação cumulativa é um fenômeno qualitativo e não redutível a uma série de equações (Argyrous e Sethi 1996: 488). Não obstante, o modelo de Setterfield que apresentamos indica a plausibilidade de modelar o conceito de causação cumulativa desenvolvido por Myrdal.

\section{Considerações Finais}

O conceito de causação cumulativa ajuda a pensar questões relacionadas ao desenvolvimento econômico, sobretudo a versão de Myrdal que apresenta uma conciliação entre Veblen e Young/Kaldor. A interpenetração entre os trabalhos de pós-keynesianos, institucionalistas e evolucionários vem ganhando adeptos, todavia ainda se encontra em estágio inicial. Esse diálogo possibilita uma abordagem plural para se pensar em desenvolvimento por incorporar tecnologia, produção, distribuição e demanda efetiva de um lado e a evolução das instituições, normas, rotinas e cultura do outro.

Desenvolvimento não é sinônimo de crescimento, constitui-se em um objetivo mais amplo no sentido de que uma sociedade que cresce menos que outra pode, contudo, desenvolver-se mais. Naturalmente, crescimento é uma peça importante do desenvolvimento e a tradição pós-keynesiana apresenta modelos interessantes nesse sentido. Por outro lado, a visão holística de desenvolvimento empreendida por Myrdal tem em seu bojo a análise de fatores históricos e institucionais que geralmente não aparecem entre os trabalhos dos pós-keynesianos. Conforme argumentamos na última seção, o conceito de causação cumulativa na esteira de Myrdal é passível de formalização a vantagem desse esforço é compreender evidências empíricas que nem sempre são explicadas por modelos que focam apenas em aspectos econômicos.

Conforme salientamos na introdução, o conceito de equilíbrio permeia a história do pensamento econômico. Vários autores o criticaram desde seu surgimento e essas críticas ecoam nos dias atuais. Embora a discussão metodológica das limitações em se pensar a economia como um sistema em equilíbrio seja da maior importância, dado a gama de autores que participaram desse debate, optamos por apresentar o conceito de causação cumulativa enquanto alternativa em vez de focar na irrelevância do conceito de equilíbrio para a ciência econômica.

Esperamos que esse artigo possa contribuir com a literatura que versa sobre desenvolvimento econômico ao reavaliar o lugar de Myrdal na história do pensamento econômico, ressaltando em que consiste sua versão de causação cumulativa e os motivos pelos quais tanto pós-keynesianos, institucionalistas e evolucionários devem revisitar Myrdal em busca de uma abordagem multidisciplinar que constitui porta de entrada para a incorporação de aspectos institucionais à análise econômica. 


\section{Referências}

ARGYROUS, G.; SETHI, R. (1996) "The theory of evolution and the evolution of theory: Veblen's methodology in contemporary perspective". Cambridge Journal of Economics, n.20, v.4, pp.475-495.

ARRIGHI, G. O. (1995) Longo século XX: dinheiro, poder e as origens de nosso tempo. Rio de Janeiro, Contraponto.

BACKHOUSE, R. B.; BOIANOVSKY, M. (2014) Transforming modern macroeconomics: exploring disequilibrium microfoundations, 1956-2003. New York, Cambridge University Press.

CORNWALL, J. (1990) The theory of economic breakdown. Oxford, Basil Blackwell. CORNWALL, J.; CORNWALL, W. (1987) "The political economy of stagnation" Journal of Economic Issues, v.21, n.2, pp.785-793.

DIXON, R.; Thirlwall, A. P. (1975) “A model of regional growth rate differences along Kaldorian lines”. Oxford Economic Papers, v. 27, n.2, pp.201-214.

FUJITA, N. (2004) "Gunnar Myrdal's theory of cumulative causation revisited" Economic Research Center, Discussion Paper n.147.

FUJITA, N. (2007) "Myrdal's Theory of Cumulative Causation". Evolutionary and Institutional Economics Review, v.3, n.2, pp.275-283

HALL, R. L.; HITCH, C. J. (1939) "Price Theory and Business Behaviour". Oxford Economic Papers, n. 2, pp. 12- 45.

HODGSON, G. M. (1989) "Institutional Rigidities and Economic Growth", Cambridge Journal of Economics, v.13, n.1, pp.79-101.

HODGSON, G. M. (1992) “Thorstein Veblen and post-Darwinian economics”. Cambridge Journal of Economics, v.16, n.3, pp.285-301.

KALDOR, N. (1935) "Market Imperfection and Excess Capacity". Economica, v. 2, n. 5, pp. 33-50.

KALDOR, N. (1966) Cases of the slow rate of economic growth of the United Kingdom. Cambridge, Cambridge University Press.

KALDOR, N. (1972) "The irrelevance of equilibrium economics". Economic Journal, v. 82, n. 328 , pp.1237-1255

KALECKI, M. (1971) Selected essays in the dynamics of the capitalist economy. Cambridge, Cambridge University Press.

LEWIN, S. B. (1996) "Economics and psychology: lessons for our own day from the early twentieth century". Journal of Economic Literature, v.34, n.3, pp.1293 1323 .

MCCOMBIE, J. S. L.; THIRLWALL, A. P. (1994) Economic Growth and the Balance of Payments Constraint. London, Macmillan.

MYRDAL, G. (1960) Teoria Econômica e Regiões Subdesenvolvidas. Rio de Janeiro MYRDAL, G. (1977) Contra a Corrente. Rio de Janeiro, Editora Campus Ltda.

MYRDAL, G. (1996 [1944]) An American Dilemma, New Brunswick, Transaction. NEALE, W. C. (1987) “Institutions”. Journal of Economic Issues, v. 21, n. 3, Evo- lutionary Economics I: Foundations of Institutional Thought, pp. 1177-1206. NELL, E. J. (1988) Prosperity and Public Spending, Boston, Unwin \& Hyman NELL, E. J. (1992) Transformational Growth and Effective Demand, London, Macmillan.

ROBINSON, J. (1953) Imperfect Competition Revisited. The Economic Journal, v. 63, n. 251, pp. 579-593.

SETTERFIELD, M. A. (1993) “A model of institutional hysteresis”. Journal of Economic Issues,v.27, n.3, pp.755-774.

SETTERFIELD, M. A. (1997a) “'History versus equilibrium' and the theory of economic growth". Cambridge Journal of Economics, v.21, n.3, pp.365-378.

SETTERFIELD, M. A. (1997b) Rapid growth and relative decline: modelling macroeconomic dynamics with hysteresis. London, Macmillan.

SMITH, A. (1996 [1776]) A Riqueza das nações: investigação sobre sua natureza e suas causas. São Paulo, Coleção Os Economistas, Abril Cultural.

SRAFFA, P. (1926) "The laws of returns under competitive conditions". Economic journal, v.36, n.144, pp.535-550.

SWEEZY, P. M. (1939) "Demand Under Conditions of Oligopoly". The Journal of Political Economy, v. 47, n. 4, pp. 568-573.

THIRLWALL, A. P. (1980) Balance of Payments Theory and UK Experience. London, Macmillan.

VEBLEN, T. (1898) “Why is economics not an evolutionary science?”. The Quarterly Journal of Economics, v.12, n.4, pp.373-397.

WALRAS, L. (1954). Elements of pure economics. Traduzido por William Jaffé, $5^{\mathrm{a}}$ edição, Londres, Allen and Unwin.

YOUNG, A. A. (1928) "Increasing returns and economic progress." The economic journal, v.38, n.152, pp.527-542. subsequente (Setterfield 1997a: 372). 